FUNCTIONAL BOWEL DISEASE

\title{
Intestinal gas distribution determines abdominal symptoms
}

\author{
H Harder, J Serra, F Azpiroz, M C Passos, S Aguadé, J-R Malagelada
}

Gut 2003;52:1708-1713

See end of article for authors' affiliations ......................

Correspondence to: Dr F Azpiroz, Digestive System Research Unit, Hospital General Vall d'Hebron, 08035Barcelona, Spain; fernando.azpiroz@wol.es

Accepted for publication 5 August 2003

\begin{abstract}
Background: Patients with functional gut disorders manifest poor tolerance to intestinal gas loads but the mechanism of this dysfunction is unknown.

Aim: Our aims were firstly, to explore the relative importance of the amount of intestinal gas versus its distribution on symptom production, and secondly, to correlate gut motility and perception of gas loads. Subjects: Fourteen healthy subjects with no gastrointestinal symptoms.

Methods: In each subject a gas mixture was infused $(12 \mathrm{ml} / \mathrm{min}$ ) either into the jejunum or rectum for one hour during blocked rectal gas outflow, and subsequently gas clearance was measured over one hour of free rectal evacuation. We measured abdominal perception, distension, and gut tone by duodenal and rectal barostats.

Results: Similar magnitude of gas retention $(720 \mathrm{ml})$ produced significantly more abdominal symptoms with jejunal compared with rectal infusion (perception score $4.4(0.4) \vee 1.5(0.5)$, respectively; $\mathrm{p}<0.01$ ) whereas abdominal distension was similar (15 (2) $\mathrm{mm}$ and 14 (1) $\mathrm{mm}$ girth increment, respectively). Jejunal gas loads were associated with proximal contraction (by $57(5) \%$ ) and colonic loads with distal relaxation (by 99 (20)\%).

Conclusion: The volume of gas within the gut determines abdominal distension whereas symptom perception depends on intraluminal gas distribution and possibly also on the gut motor response to gas loads.
\end{abstract}

$\mathrm{U}$ sing a new method to study intestinal gas dynamics and tolerance, we have shown that most healthy asymptomatic subjects tolerate large gas loads due to expeditious gas transit and evacuation. ${ }^{1}$ In contrast, when confronted with a similar challenge, patients with functional gut disorders, such as irritable bowel syndrome and functional bloating, exhibit abnormal responses, either gas retention, abdominal discomfort, or both. ${ }^{2-4}$ These data suggest that abdominal gas complaints may not be entirely explained by increased gas production but also by abnormal gas disposal. However, the intrinsic mechanism of this latter dysfunction is unknown.

The primary aim of the present study was to explore the pathogenesis of symptoms induced by intestinal gas, by comparing the relative role of two key variables in gas retention: the amount of intraluminal gas and its regional distribution. A second aim of our study was to explore the potential role of the gut motor responses to gas loads on perception. To foster these aims, we developed an experimental model of intestinal gas retention in healthy subjects whereby we infused gas either into the jejunum or rectum, and measured simultaneously abdominal perception, abdominal distension, and gut tone at both duodenal and rectal sites. As patients usually refer to gas symptoms after meals, we conducted the experiments under continuous duodenal perfusion of a fat emulsion that mimicked the postprandial caloric load. ${ }^{5}{ }^{6}$

\section{MATERIAL AND METHODS Participants}

Fourteen healthy individuals (eight woman and six men; age range 18-29 years) with no gastrointestinal complaints ${ }^{7}$ participated in the study after giving written informed consent. The protocol for the study had been previously approved by the Institutional Review Board of the University Hospital Vall d'Hebron.

\section{Gas challenge test}

Gas was infused, either into the jejunum or rectum, at $12 \mathrm{ml} /$ min using a modified volumetric pump (Asid Bonz PP 50300; Lubratronics, Unterschleissheim, Germany). The gas mixture infused (88\% nitrogen, $6.5 \%$ carbon dioxide, and $5.5 \%$ oxygen) mimicked the partial pressures of venous blood gases to minimise diffusion across the intestinal blood barrier. $^{89}$ A non-absorbable stable gaseous marker, $0.5 \%$ sulphurhexafluoride $\left(\mathrm{SF}_{6}\right)$, was added to the gas mixture. ${ }^{10} \mathrm{~A}$ lipid emulsion (Intralipid 20\%; Pharmacia and Upjohn, San Cugat del Vallés, Spain; $2 \mathrm{kcal} / \mathrm{ml}$ ) was continuously perfused at $1 \mathrm{kcal} / \mathrm{min}$ using a volumetric pump (Asid Bonz PP 50-300; Lubratronics).

The volume of gas evacuated from the rectum was measured by a barostat and recorded on a paper polygraph (model 6006; Letica, Barcelona, Spain). ${ }^{11}{ }^{12}$ A sample of gas evacuated during each 30 minute period was stored in metallised bags (gas collection $750 \mathrm{ml}$; QuinTron, Milwaukee, Wisconsin, USA) for later analysis of $\mathrm{SF}_{6}$ concentration by infrared absorbance after determination of standard curves. ${ }^{13}$

Girth was measured at 15 minute intervals by a nonstretch $48 \mathrm{~mm}$ wide belt with a metric tape measure adjusted over the umbilicus by means of two elastic bands. ${ }^{1}$

Conscious perception was measured at 15 minute intervals by means of four graphic rating scales, each graded from 0 (no perception) to 6 (painful sensation), specifically for scoring four possible abdominal sensations: (a) pressure/ bloating, (b) cramp/colicky sensation, (c) stinging sensation, and (d) other type of sensation (to be specified), respectively. The questionnaire included an additional scale to score rectal perception, and a tick box (yes/no) to signal belching. The location of the perceived sensations was also marked on an abdominal diagram. ${ }^{1-4}{ }^{15-17}$

Abbreviations: SF6, sulphurhexafluoride 


\section{Procedure}

Participants were instructed to follow a two day low flatulogenic $\operatorname{diet}^{1-4}{ }^{18}$ and were required to have one bowel movement within the 12 hours prior to the study, or otherwise the study was postponed. On the study day, participants were intubated after an eight hour fast. A tube assembly (4.9 mm od) was introduced orally and positioned under fluoroscopic control with a gas infusion channel (1.2 $\mathrm{mm}$ id) opening $5 \mathrm{~cm}$ caudal to the angle of Treitz, a cylindrical polyethylene bag $(10 \mathrm{~cm}$ long, $18 \mathrm{~cm}$ perimeter, $260 \mathrm{ml}$ capacity) in the distal duodenum, and a lipid perfusion channel (1.2 mm id) opening in the proximal duodenum. Participants were also intubated with a rectal tube assembly ( $8 \mathrm{~mm}$ od) that incorporated: (a) a gas infusion channel ( $2 \mathrm{~mm}$ id) opening at the distal tip, (b) a gas collection channel ( $3.5 \mathrm{~mm}$ id) opening by multiple side holes over the distal $3 \mathrm{~cm}$ of the tube, and (c) a polyethylene bag ( $25 \mathrm{~cm}$ perimeter, $400 \mathrm{ml}$ capacity) mounted over the tube at $5 \mathrm{~cm}$ from the tip so that the bag never collapsed the infusion and collection channels.

With subjects supine in bed at an angle of $30^{\circ}$, both duodenal and rectal bags were unfolded and connected to the respective barostat. Duodenal and rectal tone were continuously measured as volume variations at a constant pressure level of $2 \mathrm{~mm} \mathrm{Hg}$ above intra-abdominal pressure (minimal distending pressure) at each site. ${ }^{11}{ }^{14} 15$ To prevent orad pooling of secretions, the duodenal barostat was set at $0 \mathrm{~mm} \mathrm{Hg}$ pressure level for one minute at 15 minute intervals.

Duodenal lipid perfusion was started after a 15 minute baseline recording period (fig 1). After a 30 minute equilibration period, gas was infused either via the jejunal or rectal tube for 60 minutes (infusion period), during which rectal gas evacuation was blocked by a $44 \mathrm{~mm} \mathrm{Hg}$ pressure valvular system $\left(60 \mathrm{~cm} \mathrm{H} \mathrm{H}_{2} \mathrm{O}\right.$ column) applied to the collection channel. Subsequently, gas infusion was stopped, the rectal collection channel was opened, and rectal gas evacuation was measured for 60 minutes (recovery period).

\section{Experimental design}

Main study

In 10 subjects ( six women, four men), two experiments were performed in random order separated by at least a seven day interval. On one study day, gas was infused via the jejunal tube and on the other day via the rectal tube (fig l).

\section{Validation study}

The completeness of gas collection was validated by performing a washout procedure ${ }^{119}$ at the end of the main experiments as follows. In 10 subjects (in six after jejunal gas infusion and in four after rectal gas infusion) gas without $\mathrm{SF}_{6}$ was continuously infused at $30 \mathrm{ml} / \mathrm{min}$ into the jejunum for an additional 90 minute period while rectal gas evacuation

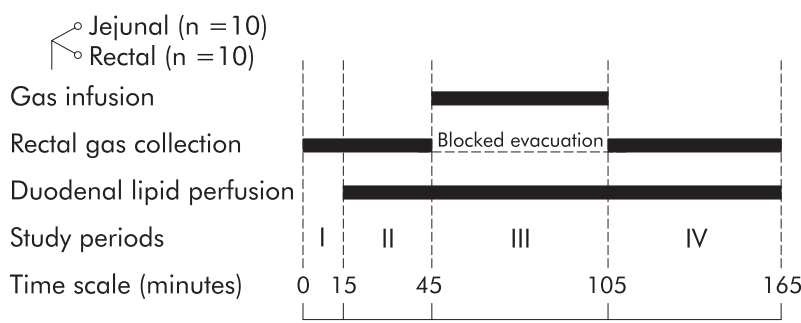

Figure 1 Methods. The study was divided in four periods: I, baseline; II, equilibration; III, infusion; and IV, recovery. During the infusion period, gas was infused ( $12 \mathrm{ml} / \mathrm{min}$ ) either into the jejunum or rectum with blocked rectal gas evacuation. During the subsequent recovery period, rectal gas was allowed to drain and gas clearance was measured. was collected. Total volume and $\mathrm{SF}_{6}$ concentration in the gas evacuated was measured to determine recovery of the $\mathrm{SF}_{6}$ infused during the experiment.

\section{Scintigraphic study}

To determine the regional distribution of the infused gas within the gut, dynamic scintigraphic imaging was performed using ${ }^{133} \mathrm{Xe}$ in four additional subjects (two women, two men). In each subject, jejunal and rectal gas infusion experiments were performed adding to the gas mixture $2 \mathrm{ml}$ ${ }^{133} \mathrm{Xe}(74 \mathrm{MBq})$ and overall following the experimental procedure and design described for the main studies. With the subject supine, anterior and posterior abdominal scans were simultaneously obtained at 60 second intervals during the 60 minute gas infusion period using a dual head large field of view gamma camera (Helix; GE-Elscint, Haifa, Israel). Due to scintigraphic imaging requirements, the procedure was simplified: perception, but not girth measurements, were taken at 15 minute intervals, and after the infusion period, the gas effluent was hermetically collected within a bag for later storage following radioprotection guidelines. The radiation exposure was less than $1.08 \mathrm{mSv}$ per experiment.

\section{Outcome measures}

The volume of gas retained within the gut was calculated as the difference between the volume of gas infused and the volume of gas recovered. Duodenal and rectal tone was measured at 15 minute intervals as the average volume recorded by the corresponding barostat over a five minute period. Scintigraphic images were analysed by a region of interest program. In each study, based on the whole sequence of images, five regions of interest were defined as the largest region excluding areas of overlap that respectively encompassed the small bowel, caecum, hepatic flexure, splenic flexure, and rectosigma. Depth corrections were performed by calculating the geometric mean of anterior and posterior scans. The activity measured in each region was divided by its surface for normalisation. In each individual scan, per cent activity in each region was expressed as per cent of total activity (sum in all five regions) at the end of the infusion period (720 $\mathrm{ml}$ gas infused).

\section{Statistical analysis}

The parameters measured during each 30 minute period were averaged in each subject and the grand mean values (SEM) in the group of subjects were calculated (median if not normally distributed data). The Komolgorov-Smirnov test was used to check the normality of data distribution. Comparisons of parametric normally distributed data were performed by the paired Student's $t$ test; otherwise the Wilcoxon signed rank test was used. The location of sensations over the abdomen and symptom distribution were compared using the $\chi^{2}$ test. Differences in xenon distribution were compared by the Wilcoxon signed rank test.

\section{RESULTS}

\section{Intraluminal gas volume and evacuation}

During the 15 minute baseline period, spontaneous evacuation of endogenous gas was very small and similar in both experimental days (76 (9) ml, pooled data). During the 30 minute equilibration period, infusion of lipids did not modify the spontaneous gas evacuation rate $(100$ (24) $\mathrm{ml}$ pooled data for both experiments; NS versus baseline period). During the 60 minute infusion period, gas evacuation was effectively blocked; only one subject had one evacuation of $30 \mathrm{ml}$ of gas associated with an intra-abdominal pressure peak above $40 \mathrm{~mm} \mathrm{Hg}$. During the 60 minute recovery period, the evacuation rate was virtually identical in 


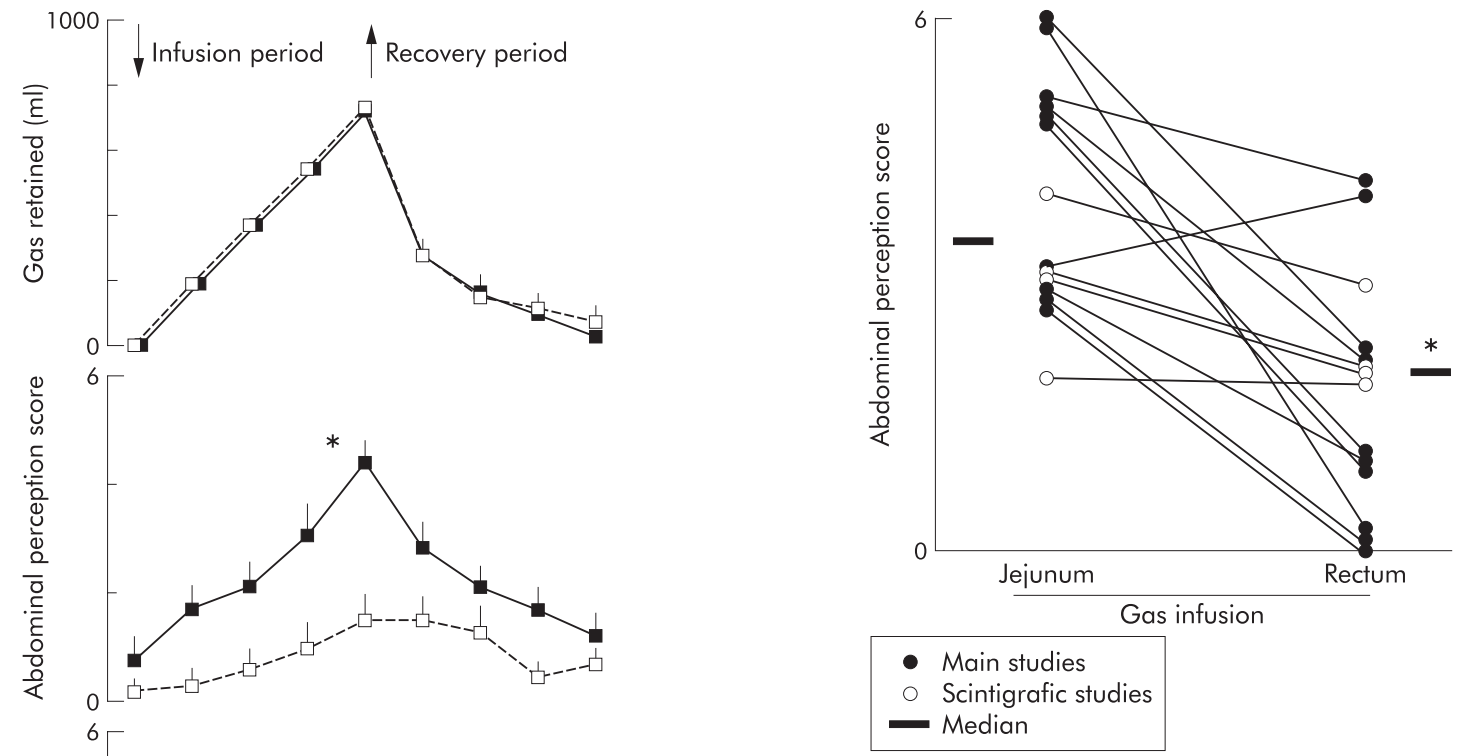

Figure 3 Effect of the gas infusion site on abdominal perception. Paired individual data (and medians) during the last 30 minutes of the jejunal and rectal gas infusion periods are shown. Note analogous perception in the main and scintigraphic studies. ${ }^{*} p<0.05$.

abdominal perception rose linearly. However, despite that, the volume of gas retained within the gut was the same regardless of the infusion site; in the same subjects, perception was significantly greater when gas was infused into the jejunum than into the rectum (figs 2,3). During the 60 minute recovery period, once the rectal collection system was open and the retained gas allowed to drain, abdominal perception in the jejunal infusion experiments rapidly decreased. In contrast, the mild perception level induced by rectal infusion of gas still persisted for 30 minutes when more than 79 (6)\% of the retained gas had been evacuated. Thereafter, perception decreased, and by the end of the 60 minute recovery period, perception reached non-significant levels both in the jejunal and rectal infusion experiments (fig 2).

The predominant abdominal symptoms were pressure/ bloating, colicky sensation, and stinging sensation, with no differences in frequency distribution in the jejunal and rectal infusion experiments (53 (13)\%, 33 (11)\%, and $14(7) \%$, respectively, during jejunal gas infusion; 43 (15)\%, 38 (15)\%, and $20(12) \%$, respectively, during rectal gas infusion; NS). During duodenal and rectal gas infusion, $55(14) \%$ and 36 (5)\% of symptoms, respectively, were referred over more than one area (NS). Symptoms were referred over the abdominal midline but the location was higher during jejunal infusion (25 (13)\% epigastric, 46 (13)\% periumbilical, and 41 (13)\% hypogastric) than during rectal infusion ( $0 \%$ epigastric, 16 (10)\% periumbilical, and 34 (14)\% hypogastric; $p<0.01$ ).

\section{Rectal perception}

During the initial baseline and equilibration periods, perception of rectal sensations was insignificant in both the jejunal and rectal infusion experiments (score $0.2(0.1)$ and $0.8(0.3)$, respectively; NS). During the gas infusion period with blocked evacuation, subjects referred rectal perception of similar intensity regardless of the gas infusion site (fig 2). During the recovery period, rectal perception decreased significantly, and by the end of 60 minutes perception had declined to non-significant levels in both experiments (fig 2). 


\section{Abdominal distension}

Girth changes during the studies paralleled the volume of gas retained in the gut. No changes were observed during the initial baseline and equilibration periods, and then girth linearly increased during the one hour infusion period. During the first 30 minutes of the recovery period, girth decreased rapidly and then more slowly during the subsequent 30 minutes. By the end of the study period, girth had returned to baseline levels. Girth changes were virtually identical in the jejunal and rectal infusion experiments (fig 2).

\section{Duodenal tone}

Baseline intra-abdominal pressure measured by the duodenal barostat was similar in the jejunal and rectal infusion experiments ( 12 (1) $\mathrm{mm} \mathrm{Hg}$; pooled data for both experiments). A distending pressure of $2 \mathrm{~mm} \mathrm{Hg}$ above this level provided a similar volume during the baseline period in both experiments (fig 4). Perfusion of duodenal lipids produced significant relaxation of the duodenum (by $33(10) \%$ at the end of the 30 minute period; $\mathrm{p}<0.05 v$ baseline). Infusion of gas into the jejunum produced the opposite effect to lipids, and duodenal tone progressively increased (by 57 (5)\% at the end of the 60 minute infusion period; $\mathrm{p}<0.05 v$ baseline and equilibration periods). During the recovery period immediately after opening the evacuation path and allowing the gas to drain, duodenal tone decreased and reached baseline levels within 15 minutes (fig 4). At the beginning of rectal gas infusion, lipid induced relaxation faded without later effects on duodenal tone (fig 4).

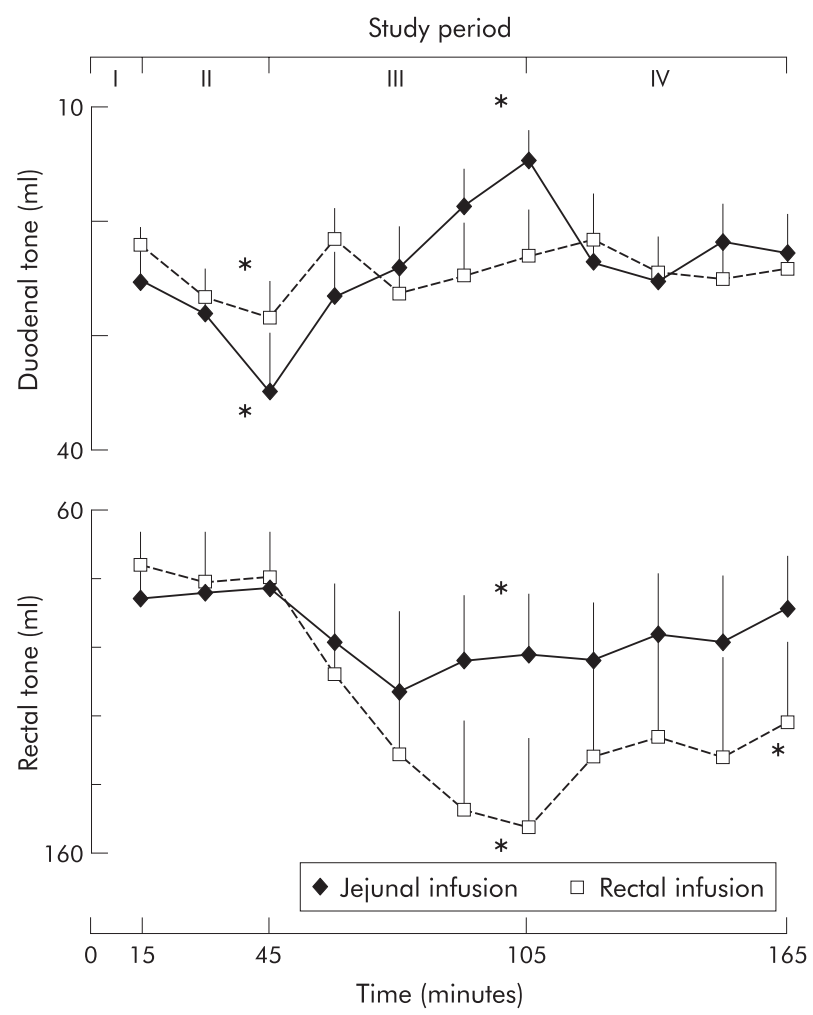

Figure 4 Effect of gas infusion into the jejunum and rectum on duodenal and rectal tone. Data during the baseline (I), equilibration (II), infusion (III), and recovery periods (IV) are shown. During the equilibration period (II), intraluminal lipids induced duodenal relaxation. During the infusion period (III), only jejunal gas infusion induced duodenal contraction whereas both jejunal and rectal gas infusion relaxed the rectum. ${ }^{*} \mathrm{p}<0.05$ versus baseline period (I).

\section{Rectal tone}

Baseline intra-abdominal pressure measured by the rectal barostat was similar in the jejunal and rectal infusion experiments (2l (1) mm Hg; pooled data for both experimental days). A distending pressure of $2 \mathrm{~mm} \mathrm{Hg}$ above this level provided a volume of air within the intrarectal bag similar in the two experimental days (fig 4). Lipid perfusion had no effects on rectal tone. Jejunal infusion of gas produced a mild but significant rectal relaxation (fig 4) that remained steady during the infusion period $(\mathrm{p}<0.05 v$ baseline period). Gas evacuation was associated with a gradual recovery of rectal tone up to a level similar to baseline. Infusion of gas into the rectum orad to the bag of the barostat produced a progressive and marked relaxation $(\mathrm{p}<0.05 v$ baseline) significantly more pronounced than during jejunal gas infusion $(\mathrm{p}<0.05$; fig 4$)$. During the recovery period, rectal tone partially recovered but a significant relaxation still persisted by the end of the study $(\mathrm{p}<0.05 v$ baseline $)$.

\section{Gas recovery (validation study)}

Recovery of $\mathrm{SF}_{6}$ after the washout performed at the end of the experiments was virtually complete (98 (1)\% and 97 (1)\% of the $\mathrm{SF}_{6}$ infused into the jejunum and into the rectum, respectively).

\section{Intestinal gas distribution (scintigraphic study)}

With ${ }^{133}$ xenon acting as a "gas marker", we observed radioscintigraphically that gas distribution within the gut was distinctively different depending on the infusion site (fig 5). During jejunal infusion, gas rapidly progressed in an aboral direction along the entire colon but as infusion progressed more gas pooled into the small bowel and caecum than into the distal colon (fig 6): at all time points, cumulative activity in the small bowel and caecum was higher than that in the rest of the colon $(p<0.05)$. In contrast, during rectal gas infusion, gas largely pooled within the distal colon (fig 6): at all time points, activity in the rectosigmoid region was higher than that in the rest of the colon $(p<0.05)$. Hence the distribution of gas in the different regions of the gut was significantly different during jejunal compared with rectal gas infusion $(\mathrm{p}<0.05)$.

Direct correlation between gas distribution and abdominal perception was obtained by measuring perception during the scintigraphic measurements: although the number of subjects was small, the results were analogous to that during the main studies (fig 3), and abdominal perception was
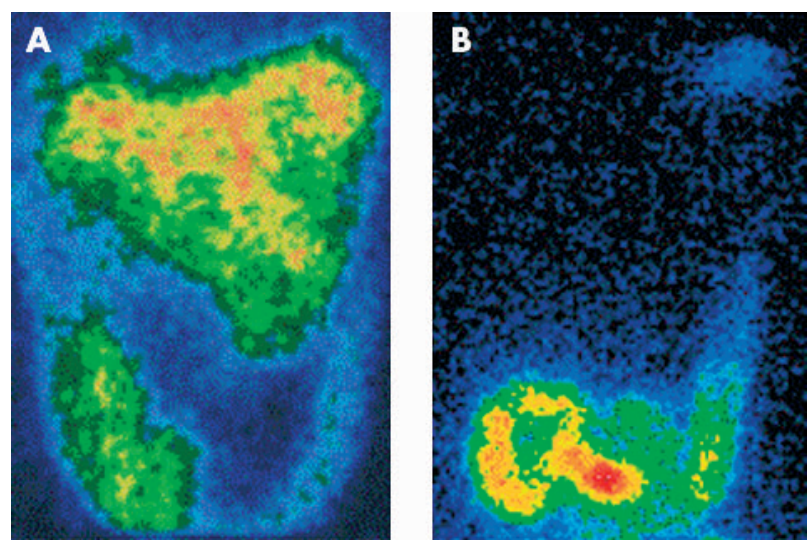

Figure 5 Scintigraphic images of ${ }^{133}$ xenon labelled gas continuously infused in the same subject for one hour $(720 \mathrm{ml})$ into the jejunum (A) and rectum (B). Note proximal distribution of jejunal versus distal distribution of rectal gas loads. 

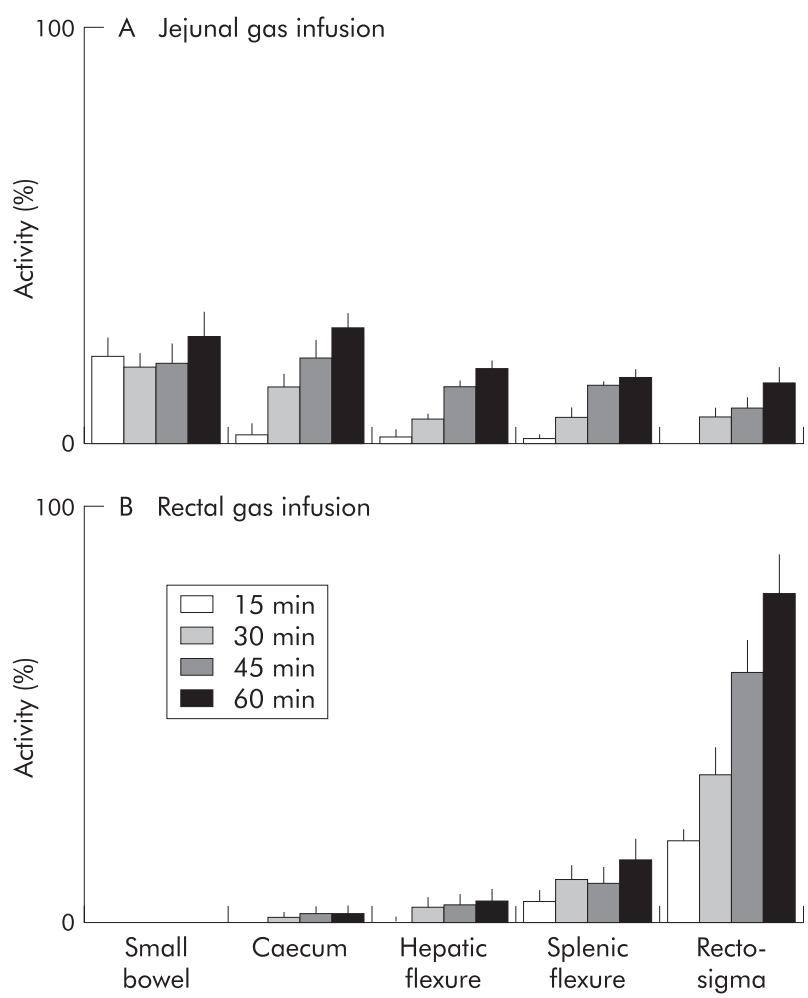

Figure 6 Scintigraphic distribution of infused gas. Per cent activity in regions of interest of ${ }^{133}$ xenon labelled gas measured by scintigraphy after 15,30,45, and 60 minutes. (A) Jejunal gas rapidly progressed in an aboral direction but tended to pool proximally in the small bowel and caecum (cumulative activity higher than in the rest of the colon; $p<0.05$ ). (B) Rectal gas largely pooled within the distal colon (activity higher than in the rest of the colon; $p<0.05$ ). Data are per cent activity in each region referred to cumulative activity in all five regions at the end of infusion (that is, $100 \%$ activity).

significantly higher during jejunal than during rectal gas infusion $(\mathrm{p}<0.05)$.

\section{DISCUSSION}

We have demonstrated that abdominal symptoms and distension associated with intestinal gas loads depend both on the total volume of retention and regional distribution. Thus abdominal discomfort is determined by the distribution of gas within the gut whereas abdominal distension is related to the absolute gas volume.

In our model, obstruction of rectal gas outflow produced similar gas retention whether gas was infused into the jejunum or rectum. However, the marked abdominal discomfort associated with jejunal gas loads suggests that the proximal bowel acts as a sensitive trigger zone capable of inducing conscious sensations, as opposed to the distal colon which appears capable of accommodating large masses of gas without discomfort. The type of symptoms induced by proximal and distal gas loads was similar but sensations were referred to a lower abdominal region in the latter case. Immediately after allowing rectal drainage, gas was cleared rapidly, at a similar rate regardless of the gas delivery site and its distribution within the gut. During the recovery period, symptoms also decreased and virtually disappeared by the end of the study. Abdominal distension paralleled gas retention, and both retention and distension were similar in the jejunal and rectal infusion experiments. Hence in contrast with perception, abdominal distension was related to the amount of gas retained in the gut, independent of its distribution.
These data from the main studies suggest but do not demonstrate that the intraluminal distribution and site of pooling differs when gas is infused into the jejunum or rectum. To obtain direct proof on the correlation between intraluminal gas distribution and symptoms, we performed an additional set of experiments using scintigraphic imaging of a gaseous marker. Due to the relatively complex set up required, these experiments only focused on intraluminal gas location and perception during jejunal and rectal infusion. Perception data reproduced the results of the main studies, and direct imaging further showed that during jejunal gas infusion, symptoms were associated with gas pooling within the proximal bowel, particularly the small intestine and caecum, but still we cannot ascertain which of these two areas acts as the trigger zone for symptoms. Probably perception mounted due to spatial summation phenomena. ${ }^{15}$ Conversely, gas infused into the rectum proved to be retained within the distal colon and associated with significantly lower perception.

Little is known of the reflex motor responses of the gut to intraluminal gas, and specifically the type of motor activity that moves gas. Conceivably, accommodation and propulsion of "low resistance" masses of gas is governed by gut capacitance, which depends on the tonic muscular activity of the gut wall (that is, gut tone). In previous studies, we observed that jejunal gas infusion is associated with duodenal contraction and rectal relaxation..$^{20}$ The present studies confirm these earlier findings, which may be part of the "law of the intestine", a general phenomenon by which the gut responds to an intraluminal load with a proximal contraction and a distal relaxation. Nevertheless, the changes observed in gut tone may be related both to gas movement and perception.

We observed that jejunal gas infusion induced significant contraction of the duodenum that increased progressively as gas continued to enter the small bowel, and paralleled the increment in perception. Conversely, when the retained gas was allowed to be expelled, both tonic contraction and symptoms declined. Interestingly, intracolonic gas during rectal infusion did not affect duodenal tone. It could be speculated that duodenal contraction in response to the jejunal gas load may operate both as a mechanism of propulsion and also as a backflow stop valve. Tonic contraction of the small bowel may have, at the same time, contributed to the production of abdominal symptoms, particularly during jejunal infusion when tonic contraction coincided with large pooling. We have previously shown that gut perception appears to be related to wall tension, rather than to intraluminal volumes, ${ }^{21}{ }^{22}$ and furthermore, discomfort associated with gas retention markedly diminishes when the gut is relaxed by prior glucagon administration. ${ }^{23}$

Relatively large gas loads directly delivered into the rectum orad to the barostat produced a marked relaxation of the rectum. Likewise, during jejunal gas infusion, small gas volumes arriving into the distal colon were associated with a significantly smaller rectal response. Furthermore, rectal relaxation increased with progressive gas pooling during rectal infusion, and decreased during gas evacuation. Hence it seems that the rectal tone response was metered depending on the amount of gas present in the distal colon, orad to the recording site. Indeed, it has been shown previously that colonic distension may induce relaxatory reflexes. ${ }^{24} 25$ This metered relaxation of the distal colon may explain why massive accommodation of rectal gas loads induced rectal sensations of similar intensity as with considerably smaller colonic gas inflow during jejunal infusion.

Our data may have important pathophysiological implications because they provide an original experimental basis to explain gas related symptoms in patients with functional gut 
disorders. Abnormal gas transit ${ }^{23}$ and possibly gas backflow ${ }^{26}$ in these patients may result in altered distribution of intestinal gas and symptoms due to proximal gut distension. Intestinal hypersensitivity, evident in proximal regions, ${ }^{7}{ }^{27-29}$ may contribute to the development of symptoms in these functional patients, even when total gas volume within the gut is not excessive.

\section{ACKNOWLEDGEMENTS}

Supported in part by the Spanish Ministry of Education (Dirección General de Enseñanza Superior del Ministerio de Educación y Cultura, BFI 2002-03413), Instituto de Salud Carlos III (grants 02/ 3036 and CO3/02), Generalitat de Catalunya (Direcció General de Recerca 1998SGR-00113), and the National Institutes of Health, USA (grant DK 57064). Dr Harder was supported by a scholarship from the Faculty of Clinical Medicine Mannheim, University of Heidelberg, Dr Serra by a scholarship from the Spanish Ministry of Health (ISC III 02/3036), and Dr Passos by a scholarship from the Brasilian Ministry of Education (Conselho Nacional de Desenvolvimento e Tecnologia). The authors thank Nuria Ferrer and Isidre Casals, Serveis Científico-Tecnics of the Central University of Barcelona, for help in gas infrared absorbance analysis; Maite Casaus and Anna Aparici for technical support; and Gloria Santaliestra for secretarial assistance.

\section{Authors' affiliations}

H Harder*, J Serra, F Azpiroz, M C Passos**, J-R Malagelada, Digestive System Research Unit, University Hospital Vall d'Hebron, Autonomous University of Barcelona, Barcelona, Spain $S$ Aguadé, Department of Nuclear Medicine, University Hospital Vall d'Hebron, Autonomous University of Barcelona, Barcelona, Spain

Present addresses:

*Department of Medicine II, Klinikum Mannheim, University of Heidelberg, Theodor-Kutzer-Ufer, 68135 Mannheim, Germany. **Instituto de Gastroenterologia, Hospital das Clinicas, Av Alfredo Baleva 110, Belo Horizonte, Minas Gerais, 30130-100 Brasil.

\section{REFERENCES}

1 Serra J, Azpiroz F, Malagelada JR. Intestinal gas dynamics and tolerance in humans. Gastroenterology 1998;115:542-50.

2 Serra J, Azpiroz F, Malagelada JR. Impaired transit and tolerance of intestinal gas in the irritable bowel syndrome. Gut $2001 ; 48: 14-19$.

3 Caldarella M, Serra J, Azpiroz F, et al. Prokinetic effects in patients with intestinal gas retention. Gastroenterology 2002;122:1748-55.

4 Serra J, Salvioli B, Azpiroz F, et al. Lipid-induced intestinal gas retention in the irritable bowel syndrome. Gastroenterology 2002;123:700-6.

5 Marsh MN, Riley SA. Digestion and absorption of nutrients and vitamins. In: Feldman M, Scharschmidt BF, Sleisenger MH, eds. Sleisenger and Fordtran's gastrointestinal and liver disease: pathophysiology/diagnosis/management. Philadelphia: WB Saunders, 1998:1471-500.

6 Hunt JN, Stubbs DF. The volume and energy content of meals as determinants of gastric emptying. J Physiol 1975;245:209-25
7 Thompson WG Longstreth G, Drossman DA, et al. Functional bowel disorders and D. Functional abdominal pain. In: Drossman DA, Corazziari E, Talley NJ, eds. The functional gastrointestinal disorders. Mc Lean: Degnon Associates, 2000:351-432.

8 Foster RE. Physiological basis of gas exchange in the gut. Ann N Y Acad Sci $1968 ; 150: 4-12$.

9 Pogrund RS, Steggerda FR. Influence of gaseous transfer between the colon and blood stream on percentage gas compositions of intestinal flatus in man. Am J Physiol 1948;153:475-82.

10 Levitt MD. Production and excretion of hydrogen gas in man. N Engl J Med 1969;281:122-7.

11 Azpiroz F, Malagelada JR. Gastric tone measured by an electronic barostat in health and postsurgical gastroparesis. Gastroenterology 1987;92:934-43

12 Azpiroz F, Malagelada JR. Physiological variations in canine gastric tone measured by an electronic barostat. Am J Physiol 1985;248:G229-37.

13 Jonmarker C, Castor R, Drefeldt B, et al. An analyzer for in-line measurement of expiratory sulfur hexafluoride concentration. Anesthesiology 1985:63:84-8

14 Rouillon JM, Azpiroz F, Malagelada JR. Sensorial and intestinointestinal reflex pathways in the human jejunum. Gastroenterology 1991;101:1606-12.

15 Serra J, Azpiroz F, Malagelada JR. Perception and reflex responses to intestinal distention in humans are modified by simultaneous or previous stimulation. Gastroenterology 1995;109:1742-9.

16 Serra J, Azpiroz F, Malagelada JR. Modulation of gut perception in humans by spatial summation phenomena. J Physiol 1998;506:579-87.

17 Gracely R. Studies of pain in normal man. In: Wall P, Melzac R, eds. Textbook of pain. Edinbourgh: Churchill Livingstone, 1994:315-36.

18 Tomlin J, Lowis C, Read NW. Investigation of normal flatus production in healthy volunteers. Gut 1991;32:665-9.

19 Levitt MD. Volume and composition of human intestinal gas determined by means of an intestinal washout technic. N Engl J Med 1971;284:1394-8.

20 Harder H, Serra J, Azpiroz F, et al. Reflex control of intestinal gas dynamics and tolerance in humans. Am J Physiol Gastrointest Liver Physiol 2003 (in press).

21 Distrutti E, Azpiroz F, Soldevilla A, et al. Gastric wall tension determines perception of gastric distention. Gastroenterology 1999;1 16:1035-42.

22 Distrutti E, Azpiroz F, Malagelada JR. Perception of rectal distension depends on wall tension, but not on expansion. Gastroenterology 1996;110:A657.

23 Serra J, Azpiroz F, Malagelada JR. Mechanisms of intestinal gas retention in humans: impaired propulsion versus obstructed evacuation. Am J Physiol Gastrointest Liver Physiol 2001;281:G138-43.

24 Sims MA, Hasler WL, Chey WD, et al. Hyperglycemia inhibits mechanoreceptor-mediated gastrocolonic responses and colonic peristaltic reflexes in healthy humans. Gastroenterology 1995; 108:350-9.

25 Biornsson ES, Chey WD, Ladabaum U, et al. Differential 5-HT3 mediation of human gastrocolonic response and colonic peristaltic reflex. Am J Physiol 1998;275:G498-505.

26 Lasser RB, Bond JH, Levitt MD. The role of intestinal gas in functional abdominal pain. N Engl J Med 1975;293:524-6.

27 Azpiroz F. Gastrointestinal perception: pathophysiological implications. Neurogastroenterol Motil 2002;14:229-39.

28 Kellow JE, Delvaux M, Azpiroz F, et al. Principles of applied neurogastroenterology: physiology/motility-sensation. In: Drossman DA, Corazziari E, Talley NJ, eds. The functional gastrointestinal disorders. Mc Lean: Degnon Associates, 2000:91-156.

29 Accarino AM, Azpiroz F, Malagelada JR. Selective dysfunction of mechanosensitive intestinal afferents in irritable bowel syndrome. Gastroenterology 1995;108:636-43. 